Background: Resting state electroencephalography (EEG) and oddball event-related potentials (ERPs) show abnormal markers in groups of amnesic mild cognitive impairment (aMCI) and Alzheimer's disease (AD) patients (Babiloni et al., 2014, Curr Alzheimer Res. 2014). This study reports the differences in these EEG/ERP markers in aMCI sub-groups classified based on "positivity" (+, prodromal AD), "intermediate level" (int.) or "negativity" (-) in CSF A $\beta 42$, A $\beta 42 /$ t-tau, and A $\beta 42 /$ p-tau. Methods: Resting state eyes-closed EEG $(\mathrm{N}=124)$ and auditory oddball ERPs $(\mathrm{N}=104)$ were recorded in aMCI subjects in the European IMI PharmaCog project (Grant Agreement $\mathrm{n}^{\circ} 115009$, www. pharmacog.org, E-ADNI). EEG markers were global power spectral density at the standard delta, theta, alpha, beta, and gamma bands. ERP marker was the greatest positive peak (about +400 $\mathrm{ms}$ after target) in posterior electrodes (P3b peak). Cortical sources of the EEG/ERP markers were estimated by eLORETA freeware. Results: The resting state EEG markers showed more active global delta sources in the MCI+ than the MCI- group in all 3 classification thresholds $(p<0.05$ corrected; Fig. 1). The ERP markers showed less active posterior parietal and cingulate sources in the MCI+ than the MCI-int and MCI- group for 1 classification threshold (A $\beta 42 /$ t-tau; $p<0.05$ corrected; Fig. 1). Conclusions: Resting state EEG rhythms and auditory oddball ERPs may reflect prodromal $\mathrm{AD}$ in a sub-group of aMCI subjects (MCI+). These markers may unveil a "neurophysiological frailty", namely abnormal mechanisms of cortical neural synchronization regulating cortical arousal in quiet wakefulness vigilance (resting state EEG) and attention/short term episodic memory demands (ERPs). Future studies should weight the contribution of the present EEG/ERP markers in the PharmaCog biomarker matrix of prodromal AD.

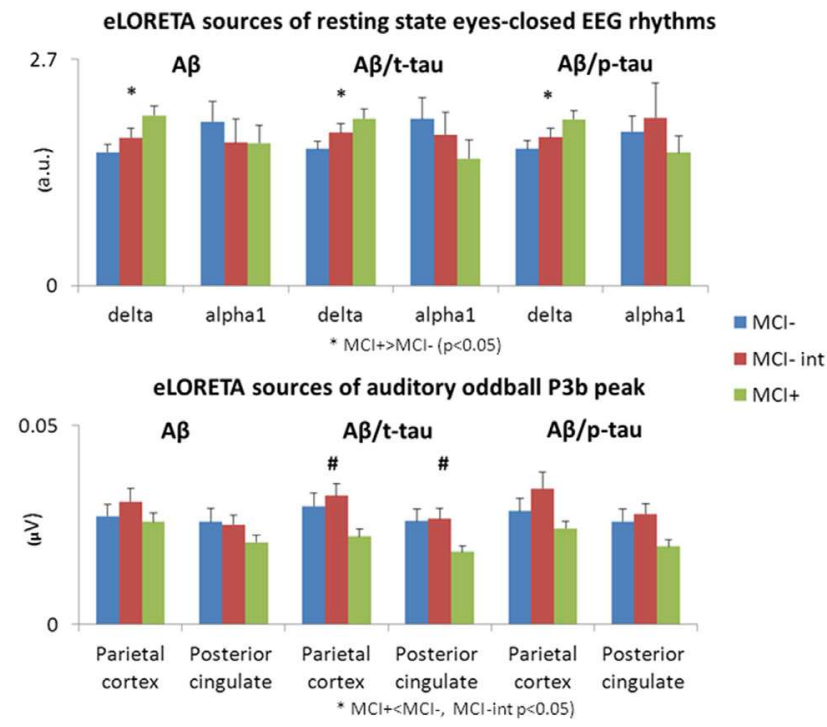

Top: Mean values ( \pm standard error, SE) of eLORETA sources of resting state eyes-closed EEG rhythms for 3 classification ( $A \beta, A \beta / p$-tau, $A \beta / p$ tau) for the factors: (i) Group (MCI-, MCI- int, MCI+) and (ii) Band (delta, alpha 1). Bottom: Mean values ( \pm SE) of eLORETA sources of auditory oddball P3b peak for 3 classification ( $A \beta, A \beta / t$-tau, $A \beta / p$-tau) for the factors: (i) Group (MCI-, MCI- int, MCI+) and (ii) Region of interest (parietal cortex, posterior cingulate cortex).

\section{P3-058 AT REST EEG SPECTRA POWER CORRELATES OF MEMORY PERFORMANCE OF OLDER AFRICAN AMERICANS ENDORSING SUBJECTIVE MEMORY COMPLAINTS}

Voyko Kavcic ${ }^{1}$, Kacy Davis ${ }^{1}$, Bruno Giordani ${ }^{2},{ }^{1}$ Wayne State University, Detroit, MI, USA; ${ }^{2}$ University of Michigan, Ann Arbor, MI, USA. Contacte-mail:eq2623@wayne.edu

Background: Frequently used criteria to identify elderly at risk for cognitive decline and dementia is presence of a subjective memory complaints (SMC). Here we report the correlations of persons with SMC between their memory performance as measured by accuracy scores and diffusion model parameters (calculated from the accuracy score, mean response time, and response variability) on the One Card Learning (OCL) subtest from computerized CogState Battery and their at-rest, eyes-closed, EEG spectral power. Methods: Data were collected from 30 community-dwelling African American volunteers ( 25 females, 5 males) with SMC, age $\geq 65$ years, mean MMSE $=28.32$, range 25-30. We correlated the OCL accuracy and diffusion model parameters (drift rate, boundary, non-decision time) with several, at-rest with eyes closed, EEG spectral measures: absolute power from all major frequency bands $(\theta, \delta, \alpha$, and $\beta$ ), relative power in delta and theta bands, and peak alpha frequency and amplitude. Results: Results showed that OCL accuracy was significantly, negatively correlated with increased absolute and relative EEG delta power in central, parietal, and occipital ROIs. We also observed significant correlations between diffusion model drift rate parameters and EEG spectral power. Specifically, the higher delta power from central, parietal, and occipital ROIs were significantly correlated with lower drift rate (i.e., slower accumulation of information needed for decision making). Lower drift rate also was significantly, negatively correlated with increased peak alpha amplitude at frontal, left temporal, and parietal ROIs. There were also significant correlations between the diffusion model boundary parameter and peak alpha amplitude at occipital ROI and relative theta power at right temporal ROI. Conclusions: The findings of the present study show that EEG spectal powers of lower frequency bands are related to lower mnemonic performance in older African Americans who endorse SMC. In addition, increased delta and theta powers in the intrinsic, default mode brain state could be conceptualized as reflecting a compensatory mechanism in an already compromised brain. Results support the use of easily accessible, cost-effective, culture-fair, readily acceptable, noninvasive EEG recordings that can provide reliable diagnostic (and potentially prognostic) tools for differentiation of at risk from stable older African Americans within a community setting.

\section{P3-059 DOES SYNTHETIC DATA OVERSAMPLING IN FEATURE SELECTION IMPROVE THE CLASSIFICATION RATE IN ALZHEIMER'S DISEASE?}

Yuri Elias Rodrigues ${ }^{1}$, Evandro Manica ${ }^{1}$, Eduardo R. Zimmer ${ }^{1}$, Eliete Biasotto Hauser ${ }^{2}$, Pedro Rosa-Neto ${ }^{3,4}$ and Alzheimer's Team, ${ }^{1}$ Federal University of Rio Grande dos Sul, Porto Alegre, Brazil; ${ }^{2}$ Catholic University of Rio Grande do Sul, Porto Alegre, Brazil; ${ }^{3} \mathrm{McC}$ Connell Brain Imaging Centre, Montréal, QC, Canada; ${ }^{4}$ McGill University Research Centre for Studies in Aging, Verdun, QC, Canada. Contact e-mail: yuri. rodrigues@acad.pucrs.br

Background:Large-scale trials are sampled with unequal proportions since the complex pathological conformations have its own most probability of occurrence. This produces 